\title{
Hospitalizations of Children and Adolescents with Laboratory-Confirmed COVID-19 - COVID-NET, 14 States, July 2021-January 2022
}

\begin{abstract}
Kristin J. Marks, $\mathrm{PhD}^{1,2}$; Michael Whitaker, $\mathrm{MPH}^{1}$; Onika Anglin, $\mathrm{MPH}^{1,3}$; Jennifer Milucky, MSPH${ }^{1}$; Kadam Patel, $\mathrm{MPH}^{1,3}$; Huong Pham, MPH ${ }^{1}$; Shua J. Chai, MD ${ }^{4,5}$; Pam Daily Kirley, MPH4 ; Isaac Armistead, MD ${ }^{6}$; Sarah McLafferty, MPH6; James Meek, MPH7 Kimberly Yousey-Hindes, $\mathrm{MPH}^{7}$; Evan J. Anderson, $\mathrm{MD}^{8,9,10}$; Kyle P. Openo, $\mathrm{DrPH}^{8,9}$; Andy Weigel, MSW ${ }^{11}$; Justin Henderson, $\mathrm{MPH}^{12}$; Val Tellez Nunez, MPH ${ }^{12}$; Kathryn Como-Sabetti, MPH ${ }^{13}$; Ruth Lynfield, MD ${ }^{13}$; Susan L. Ropp, PhD ${ }^{14}$; Chad Smelser, MD ${ }^{14}$; Grant R. Barney, MPH ${ }^{15}$; Alison Muse, MPH ${ }^{15}$; Nancy M. Bennett, MD ${ }^{16}$; Sophrena Bushey, MHS ${ }^{16}$; Laurie M. Billing, MPH ${ }^{17}$; Eli Shiltz, MPH ${ }^{17}$; Nasreen Abdullah, MD ${ }^{18}$; Melissa Sutton, MD ${ }^{18}$; William Schaffner, MD ${ }^{19}$; H. Keipp Talbot, MD ${ }^{19}$; Ryan Chatelain, $\mathrm{MPH}^{20}$; Andrea George, MPH ${ }^{20}$; Christopher A. Taylor, $\mathrm{PhD}^{1}$; Meredith L. McMorrow, $\mathrm{MD}^{1}$; Cria G. Perrine, PhD ${ }^{1}$; Fiona P. Havers, $\mathrm{MD}^{1}$; COVID-NET Surveillance Team
\end{abstract}

On February 15, 2022, this report was posted as an MMWR Early Release on the MMWR website (https://www.cdc.gov/mmwr).

The first U.S. case of COVID-19 attributed to the Omicron variant of SARS-CoV-2 (the virus that causes COVID-19) was reported on December 1, 2021 (1), and by the week ending December 25, 2021, Omicron was the predominant circulating variant in the United States.* Although COVID-19-associated hospitalizations are more frequent among adults, ${ }^{\dagger}$ COVID-19 can lead to severe outcomes in children and adolescents (2). This report analyzes data from the Coronavirus Disease 19-Associated Hospitalization Surveillance Network (COVID-NET) ${ }^{\S}$ to describe COVID-19-associated hospitalizations among U.S. children (aged 0-11 years) and adolescents (aged 12-17 years) during periods of Delta (July 1-December 18, 2021) and Omicron (December 19, 2021-January 22, 2022) predominance. During the Delta- and Omicron-predominant periods, rates of weekly COVID-19-associated hospitalizations per 100,000 children and adolescents peaked during the weeks ending September 11, 2021, and January 8, 2022, respectively. The Omicron variant peak $(7.1$ per 100,000$)$ was four times that of the Delta variant peak (1.8), with the largest increase observed among children aged 0-4 years. 9 During December 2021, the monthly hospitalization rate among unvaccinated adolescents aged 12-17 years (23.5) was six times that among fully vaccinated adolescents (3.8). Strategies to prevent COVID-19 among children and adolescents, including vaccination of eligible persons, are critical.**

COVID-NET conducts population-based surveillance for laboratory-confirmed COVID-19-associated hospitalizations

\footnotetext{
* https://covid.cdc.gov/covid-data-tracker/\#variant-proportions

$\dagger$ https://www.cdc.gov/coronavirus/2019-ncov/covid-data/investigationsdiscovery/hospitalization-death-by-age.html

$\$$ https:/www.cdc.gov/coronavirus/2019-ncov/covid-data/covid-net/purposemethods.html

9 COVID-NET hospitalization data are preliminary and subject to change as more data become available. In particular, case counts and rates for recent hospital admissions are subject to lag.

** https://www.cdc.gov/coronavirus/2019-ncov/vaccines/your-vaccination.html
}

in 99 counties across 14 states. $^{\dagger \dagger}$ Among residents of a predefined surveillance catchment area, COVID-19-associated hospitalizations are defined as receipt of a positive SARS-CoV-2 real-time reverse transcription-polymerase chain reaction (RT-PCR) or rapid antigen detection test result during hospitalization or during the 14 days before admission. This analysis describes weekly hospitalization rates during the weeks ending July 3, 2021-January 22, 2022, to coincide with a period during which detailed clinical data (e.g., intensive care unit [ICU] admission) were available (monthly, July 1-December 31, 2021). Unadjusted weekly COVID-19-associated hospitalization rates were calculated by dividing the total number of hospitalized patients by the population estimates within each age group for the counties included in the surveillance catchment area. ${ }^{\$ S}$ ICU admission rates were similarly calculated using 2-week periods. All rates were estimated per 100,000 population for children, adolescents, or both.

Among adolescents aged 12-17 years, hospitalization rates were calculated by COVID-19 vaccination status, which was determined both for hospitalized patients and the catchment population using linkage to state immunization information systems data. 99 Monthly incidence was calculated by summing the total number of hospitalized adolescents who were fully vaccinated ( $\geq 14$ days after final dose in primary series) for each day of the month and dividing by the sum of fully vaccinated adolescents in the underlying population for each day of the month;

\footnotetext{
$\dagger \dagger$ California, Colorado, Connecticut, Georgia, Iowa, Maryland, Michigan, Minnesota, New Mexico, New York, Ohio, Oregon, Tennessee, and Utah.

$\$ \$$ Rates are calculated using the National Center for Health Statistics vintage 2020 bridged-race postcensal population estimates for the counties included in surveillance (https://www.cdc.gov/nchs/nvss/bridged_race.htm).

99 The Food and Drug Administration granted emergency use authorization for the Pfizer-BioNTech COVID-19 vaccine for adolescents aged 12-15 years on May 10, 2021. The earliest date that adolescents in this age group could have met the definition for being a fully vaccinated COVID-19 patient was June 14, 2021.
} 
the same method was used to calculate incidence in unvaccinated adolescents.*** Rate ratios (RRs) and 95\% CIs were calculated.

Trained surveillance staff members conducted medical chart abstractions for all pediatric COVID-NET patients using a standardized case report form through November 2021. Because of the large number of cases during December 2021, some sites examined clinical outcome data on a representative sample of hospitalized children. ${ }^{\dagger \dagger}$ Data on indicators of severe disease were collected (i.e., hospital length of stay, ICU admission, use of invasive mechanical ventilation [IMV], ${ }^{\$ S \$}$ and in-hospital death), as were data on primary reason for admission 999 and symptoms that were present when the patient was admitted ${ }^{* * * *}(3)$. Proportions were compared between periods of Delta predominance (July 1-December 18, 2021) and Omicron predominance (December 19-31, 2021); a variant that accounted for $>50 \%$ of sequenced isolates was considered to be predominant. ${ }^{\dagger \dagger \dagger}$ A similar analysis was

*** Fully vaccinated adolescents with COVID-19-associated hospitalizations were defined as those who had received the final dose in their primary series $\geq 14$ days before receiving a positive SARS-CoV- 2 test result associated with their hospitalization. Adolescents who received only 1 vaccine dose $\geq 14$ days before the SARS-CoV-2 test date or had received a single dose of vaccine $<14$ days before the positive SARS-CoV-2 test results were considered partially vaccinated; they were not included in rates and were grouped with unvaccinated adolescents in other analyses. Unvaccinated adolescents were defined as those who did not meet the criteria for being fully or partially vaccinated. Additional COVID-NET methods for determining vaccination status have been described previously (https://www.medrxiv.org/cgi/ content/short/2021.08.27.21262356v1).

it† Colorado, Georgia, New Mexico, and Utah sampled $50 \%$ of patients during the month of December. All other sites included $100 \%$ of cases. To produce random samples of hospitalized patients for medical record abstraction, random numbers (1-100) are automatically generated and assigned to each patient as their data are entered into the surveillance database. Percentages are weighted to account for the probability of selection for sampled patients.

$\$ \mathbb{S} \mathbb{S}$ ICU admission and IMV are not mutually exclusive categories, and patients could have received both.

999 Among sampled patients, COVID-NET collects data on the primary reason for admission to differentiate hospitalizations of patients with laboratoryconfirmed SARS-CoV-2 infection who are likely admitted primarily for COVID-19 illness rather than for other reasons. During chart review, if the surveillance officer finds that the chief complaint or history of present illness mentions fever/respiratory illness, COVID-19-like illness, or a suspicion for COVID-19, then the case is categorized as COVID-19 related illness as the primary reason for admission.

**** COVID-19-related symptoms included respiratory symptoms (congestion or runny nose, cough, hemoptysis or bloody sputum, shortness of breath or respiratory distress, sore throat, upper respiratory infection, influenza-like illness, and wheezing) and nonrespiratory symptoms (abdominal pain, altered mental status or confusion, anosmia or decreased smell, chest pain, conjunctivitis, diarrhea, dysgeusia or decreased taste, fatigue, fever or chills, headache, muscle aches or myalgias, nausea or vomiting, rash, and seizures), and among those aged $<2$ years, included apnea, cyanosis, decreased vocalization or stridor, dehydration, hypothermia, inability to eat or poor feeding, and lethargy. Symptoms are abstracted from the medical chart and might not be complete.

itt† Delta became the predominant $(>50 \%)$ variant circulating in the United States the week ending July 3, 2021. By the week ending December 18, 2021 , Omicron accounted for $38 \%$ of circulating variants; Omicron became the predominant variant the week ending December 25 at $74 \%$. completed by vaccination status among adolescents, the only pediatric age group for whom a COVID-19 vaccine had been approved throughout the surveillance period. Wilcoxon ranksum tests were used to compare medians, and chi-square or Fisher's exact tests were used to compare proportions; p-values $<0.05$ were considered statistically significant. Percentages were weighted to account for the probability of selection for sampled cases and further adjusted to account for nonresponse (defined as an incomplete chart review). Data were analyzed using SAS (version 9.4; SAS Institute). This activity was reviewed by CDC and was conducted consistent with applicable federal law and CDC policy. ${ }^{\$ S \$ S}$

During the Delta- and Omicron-predominant periods, pediatric weekly hospitalization rates peaked during the weeks ending September 11, 2021, and January 8, 2022, respectively; the Omicron variant peak (7.1 per 100,000 children and adolescents) was four times that of the Delta variant peak (1.8). Hospitalization rates among children aged $0-4$ years were approximately five times as high during the peak week of the Omicron period (15.6) than during the Delta period (2.9) $(\mathrm{RR}=5.4 ; 95 \% \mathrm{CI}=4.0-7.2)$ (Figure); $\mathrm{RRs}$ were also increased among children aged $5-11$ years $($ Delta $=1.1$; Omicron $=2.4$; $\mathrm{RR}=2.3 ; 95 \% \mathrm{CI}=1.5-3.6)$ and adolescents aged $12-17$ years $($ Delta $=1.7 ;$ Omicron $=5.9 ; \mathrm{RR}=3.5 ; 95 \% \mathrm{CI}=2.5-5.0)$. Peak ICU admission rates for children and adolescents were 1.4 times higher during Omicron predominance (2-week period ending December 31, 2021 [1.5]) than during Delta predominance (2-week period ending September 11, 2021 [1.1]). During December 2021, when both variants were circulating, the rates of hospitalization were 23.5 and 3.8 per 100,000 among unvaccinated and fully vaccinated adolescents, respectively $(\mathrm{RR}=6.3 ; 95 \% \mathrm{CI}=4.4-8.6)$.

Complete clinical data were available for 1,8349999 and $266^{* * * * *}$ hospitalized children and adolescents in the Deltapredominant (July 1-December 18, 2021) and Omicronpredominant (December 19, 2021-December 31, 2021) periods, respectively. The proportions of hospitalized children and adolescents requiring ICU admission (Delta $=27.8 \%$; Omicron $=20.2 \%)$ or IMV (Delta $=6.3 \%$; Omicron $=2.3 \%)$ were significantly lower during the Omicron period (Table 1). No significant difference was detected between the Delta- and Omicron-predominant periods in the proportion of patients

\footnotetext{
\$SS\$ 45 C.F.R. part 46.102(l)(2), 21 C.F.R. part 56; 42 U.S.C. Sect. 241(d); 5 U.S.C. Sect. 552a; 44 U.S.C. Sect. 3501 et seq.

9999 Among the 1,943 sampled children and adolescents with COVID-19associated hospitalizations during July 1-December 18, 2021, a total of 1,834 (94.4\%) had data available on hospital length of stay, ICU admission, receipt of IMV, and in-hospital death at the time of reporting.

***** Among the 281 sampled children and adolescents with COVID-19associated hospitalizations during December 19-31, 2021, a total of 266 $(94.7 \%)$ had data available on hospital length of stay, ICU admission, receipt of IMV, and in-hospital death at the time of reporting.
} 
FIGURE. Weekly COVID-19-associated hospitalization rates* among children and adolescents aged 0-17 years, by age group - COVID-NET, 14 states, ${ }^{\dagger}$ July 3, 2021-January 22, 2022

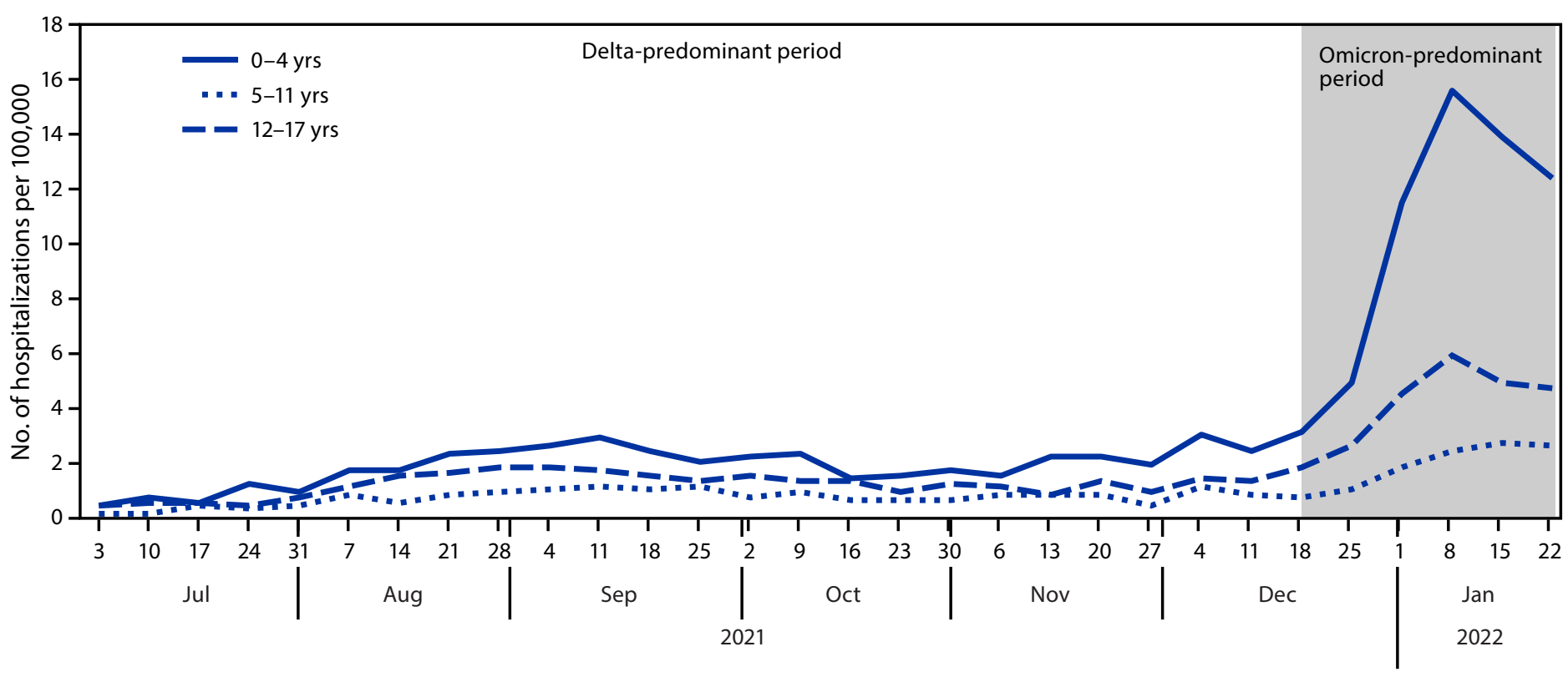

Surveillance week end date

Abbreviation: COVID-NET = Coronavirus Disease 2019-Associated Hospitalization Surveillance Network.

* Number of patients with laboratory-confirmed COVID-19-associated hospitalizations per 100,000 population; rates are subject to change as additional data are reported. + COVID-NET sites are in the following 14 states: California, Colorado, Connecticut, Georgia, lowa, Maryland, Michigan, Minnesota, New Mexico, New York, Ohio, Oregon, Tennessee, and Utah. Starting the week ending December 4, 2021, Maryland data are removed from weekly rate calculations.

with COVID-19-related symptoms recorded at admission $(87.7 \%$ versus $86.9 \%)$ or with COVID-19 as the primary reason for admission $(81.3 \%$ versus $81.6 \%)$.

The proportion of hospitalized adolescents who were fully vaccinated was significantly lower during the Deltapredominant period $(8.3 \%)$ than during the Omicronpredominant period $(22.2 \%)$ (Table 1$)$, consistent with increasing adolescent vaccination coverage during the surveillance period. During July 1-December 31, 2021, 42.4\% of hospitalized unvaccinated adolescents were non-Hispanic Black adolescents (Table 2). A higher proportion of unvaccinated adolescents $(70.3 \%)$ than fully vaccinated adolescents (40.8\%) had COVID-19 as a primary reason for admission. A significantly higher proportion of unvaccinated adolescents were admitted to the ICU (30.3\%) than were those who were vaccinated $(15.5 \%)$.

\section{Discussion}

The Omicron variant, which is associated with increased transmissibility and partial escape from infection- or vaccineinduced immunity, replaced Delta as the predominant variant in the United States in late December 2021 (1). Once the Omicron variant became predominant, peak population-based COVID-19-associated hospitalization rates among children and adolescents were four times as high as rates during the peak of the Delta period. Children aged 0-4 years, who were ineligible for vaccination during this time, experienced the largest increase in hospitalization rates. Observed indicators of severe COVID-19 among children and adolescents, in addition to the potential for longer-term sequelae $(4,5)$, highlight the importance of multicomponent strategies to reduce the incidence of COVID-19, including vaccination of eligible persons and other prevention measures. ${ }^{\dagger \dagger \dagger \dagger}$

Among adolescents aged 12-17 years, the only pediatric age group for whom a COVID-19 vaccine was approved throughout the study period, December hospitalization rates among unvaccinated adolescents were approximately six times those among fully vaccinated adolescents, suggesting that vaccines were highly effective in preventing serious COVID-19 illness. Vaccination eligibility was expanded to include children aged 5-11 years on November 2, 2021. As of December 31, 2021, $54 \%$ of the population aged $12-17$ years and $16 \%$ of those aged 5-11 years had completed a COVID-19 primary vaccination series. $\$ \$ \$ \$ \$$ Increasing vaccination coverage among both age groups can reduce COVID-19-associated hospitalizations (G);

†††† https://www.cdc.gov/vaccines/hcp/acip-recs/vacc-specific/covid-19.html $\$ \$ \Phi \$ \$$ https://covid.cdc.gov/covid-data-tracker/\#vaccination-demographics-trends 
TABLE 1. Demographic and clinical characteristics and outcomes among children and adolescents aged 0-17 years with laboratory-confirmed COVID-19-associated hospitalizations, ${ }^{*}$ by date of admission - COVID-NET, 14 states, ${ }^{\dagger}$ July 1-December 31, 2021

\begin{tabular}{|c|c|c|c|c|}
\hline \multirow[b]{3}{*}{ Characteristic } & \multicolumn{3}{|c|}{ No. of hospitalized children (\%) } & \multirow[b]{3}{*}{ p-value ${ }^{\S}$} \\
\hline & Total & & & \\
\hline & Jul 1-Dec 31 & Jul 1-Dec 18 & Dec 19-31 & \\
\hline Total & $2,100(100.0)^{\Upsilon}$ & $1,834(82.3)^{9}$ & $266(17.7)^{9}$ & - \\
\hline Age, yrs, median (IQR) & $7(1-14)$ & $7(1-14)$ & $3.5(0.4-13)$ & $<0.001$ \\
\hline $\begin{array}{l}\text { Age group, yrs } \\
0-4 \\
5-11 \\
12-17\end{array}$ & $\begin{array}{l}920(44.6) \\
460(21.5) \\
720(33.9)\end{array}$ & $\begin{array}{l}778(42.5) \\
417(22.5) \\
639(34.9)\end{array}$ & $\begin{array}{r}142(54.2) \\
43(16.9) \\
81(28.9)\end{array}$ & 0.003 \\
\hline $\begin{array}{l}\text { Sex } \\
\text { Male } \\
\text { Female }\end{array}$ & $\begin{array}{l}1,081(51.7) \\
1,019(48.3)\end{array}$ & $\begin{array}{l}934(51.2) \\
900(48.8)\end{array}$ & $\begin{array}{l}147(54.2) \\
119(45.8)\end{array}$ & 0.38 \\
\hline $\begin{array}{l}\text { Race and ethnicity** } \\
\text { Hispanic } \\
\text { Black, non-Hispanic } \\
\text { White, non-Hispanic } \\
\text { Asian or Pacific Islander, non-Hispanic } \\
\text { All other races }{ }^{\dagger \dagger} \\
\text { Unknown race and ethnicity }\end{array}$ & $\begin{array}{r}463(21.8) \\
736(35.8) \\
670(31.3) \\
82(3.9) \\
47(2.3) \\
102(5.0)\end{array}$ & $\begin{array}{r}420(23.1) \\
619(33.4) \\
598(32.6) \\
71(3.9) \\
41(2.3) \\
85(4.8)\end{array}$ & $\begin{array}{r}43(15.7) \\
117(47.1) \\
72(25.5) \\
11(3.7) \\
6(2.1) \\
17(5.9)\end{array}$ & $<0.001$ \\
\hline $\begin{array}{l}\text { Primary reason for admission } \$ \S \\
\text { Likely related to COVID-19 } \\
\text { Obstetrics } \\
\text { Inpatient surgery } \\
\text { Psychiatric admission requiring medical care } \\
\text { Trauma } \\
\text { Other reason } \\
\text { Unknown reason }\end{array}$ & $\begin{array}{r}1,703(81.3) \\
63(2.9) \\
53(2.6) \\
118(5.6) \\
75(3.5) \\
78(3.8) \\
6(0.3)\end{array}$ & $\begin{array}{r}1,489(81.3) \\
57(3.0) \\
43(2.5) \\
108(5.9) \\
67(3.7) \\
62(3.3) \\
6(0.3)\end{array}$ & $\begin{array}{r}214(81.6) \\
6(2.2) \\
10(3.3) \\
10(4.0) \\
8(2.8) \\
16(6.1) \\
0(-)\end{array}$ & 0.19 \\
\hline $\begin{array}{l}\text { COVID-19-related symptoms at admission } \\
\text { Yes } \\
\text { No }\end{array}$ & $\begin{array}{r}1,832(87.6) \\
264(12.4)\end{array}$ & $\begin{array}{r}1,604(87.7) \\
228(12.3)\end{array}$ & $\begin{array}{r}228(86.9) \\
36(13.1)\end{array}$ & 0.72 \\
\hline $\begin{array}{l}\text { Hospitalization outcomes } \\
\text { Length of hospital stay, days, median (IQR) } \\
\text { ICU admission*** } \\
\text { Invasive mechanical ventilation*** } \\
\text { In-hospital death }\end{array}$ & $\begin{array}{r}3(1-5) \\
562(26.4) \\
118(5.6) \\
11(0.5)\end{array}$ & $\begin{array}{r}3(2-5) \\
510(27.8) \\
112(6.3) \\
11(0.6)\end{array}$ & $\begin{array}{r}2(1-5) \\
52(20.2) \\
6(2.3) \\
0(-)\end{array}$ & $\begin{array}{l}0.15 \\
0.01 \\
0.01 \\
0.38\end{array}$ \\
\hline $\begin{array}{l}\text { Vaccination status (among patients aged } 12 \\
\text { Fully vaccinated } \\
\text { Unvaccinated }\end{array}$ & $\begin{array}{r}71(9.9) \\
647(90.1)\end{array}$ & $\begin{array}{r}53(8.3) \\
584(91.7)\end{array}$ & $\begin{array}{l}18(22.2) \\
63(77.8)\end{array}$ & $<0.001$ \\
\hline
\end{tabular}

See table footnotes on the next page.

enhanced outreach strategies are needed to address disparities in vaccination coverage by race/ethnicity.

Consistent with national hospital surveillance data (7), the findings in this report indicate that the Omicron-predominant period had higher rates of pediatric COVID-19 hospitalizations than the Delta-predominant period. No differences were found between the Delta- and Omicron-predominant periods in the proportion of hospitalizations that were likely to be related to COVID-19. Findings suggest that incidental admissions do not account for the increase in hospitalization rates observed during the Omicron period. Throughout the COVID-19 pandemic, admissions for reasons other than COVID-19 have been recorded $(8,9)$, and during both the Delta- and Omicron-predominant periods, incidental admissions were more likely among fully vaccinated adolescents.
Reasons for admission should continue to be monitored as more children and adolescents become fully vaccinated.

The findings in this report are subject to at least six limitations. First, COVID-19-associated hospitalizations might have been missed because of testing practices and test availability. Second, the period of Omicron variant predominance with available detailed clinical data is brief (December 19-31, 2021) and does not capture the peak of hospitalizations during the Omicron period; in addition, the Delta variant was still circulating in late December. Third, accounting for seasonality in comparisons of Delta and Omicron predominant periods was not possible. Fourth, the number of hospitalized children eligible for vaccination remained low at the time of reporting, and hospitalization rates stratified by vaccination status are subject to error if misclassification of vaccination status occurred. Fifth, because children aged 5-11 years could not meet the 
TABLE 1. (Continued) Demographic and clinical characteristics and outcomes among children and adolescents aged 0-17 years with laboratoryconfirmed COVID-19-associated hospitalizations, ${ }^{*}$ by date of admission - COVID-NET, 14 states, ${ }^{\dagger}$ July 1-December 31,2021

\begin{abstract}
Abbreviations: COVID-NET = Coronavirus Disease 2019-Associated Hospitalization Surveillance Network; ICU = intensive care unit.
* Data are from a weighted sample of hospitalized children and adolescents with completed medical record abstractions. Sample sizes presented are unweighted with weighted percentages.

${ }^{\dagger}$ Includes persons admitted to a hospital with an admission date during July 1-December 31, 2021. Maryland contributed data through November 26, 2021. Counties included in COVID-NET surveillance: California (Alameda, Contra Costa, and San Francisco counties); Colorado (Adams, Arapahoe, Denver, Douglas, and Jefferson counties); Connecticut (Middlesex and New Haven counties); Georgia (Clayton, Cobb, DeKalb, Douglas, Fulton, Gwinnett, Newton, and Rockdale counties); lowa (one county represented); Maryland (Allegany, Anne Arundel, Baltimore, Baltimore City, Calvert, Caroline, Carroll, Cecil, Charles, Dorchester, Frederick, Garrett, Harford, Howard, Kent, Montgomery, Prince George's, Queen Anne's, St. Mary's, Somerset, Talbot, Washington, Wicomico, and Worcester counties); Michigan (Clinton, Eaton, Genesee, Ingham, and Washtenaw counties); Minnesota (Anoka, Carver, Dakota, Hennepin, Ramsey, Scott, and Washington counties); New Mexico (Bernalillo, Chaves, Doña Ana, Grant, Luna, San Juan, and Santa Fe counties); New York (Albany, Columbia, Genesee, Greene, Livingston, Monroe, Montgomery, Ontario, Orleans, Rensselaer, Saratoga, Schenectady, Schoharie, Wayne, and Yates counties); Ohio (Delaware, Fairfield, Franklin, Hocking, Licking, Madison, Morrow, Perry, Pickaway, and Union counties); Oregon (Clackamas, Multnomah, and Washington counties); Tennessee (Cheatham, Davidson, Dickson, Robertson, Rutherford, Sumner, Williamson, and Wilson counties); and Utah (Salt Lake County).

$\S$ Proportions between the Delta and Omicron predominance periods were compared with chi-square tests or Fisher's exact tests (as appropriate), and medians were compared with the Wilcoxon rank-sum test; $p$-values $<0.05$ were considered statistically significant.

" Data are missing for $<5 \%$ of observations for all variables.

** If ethnicity was unknown, non-Hispanic ethnicity was assumed.

$\mathrm{t}^{+}$Includes non-Hispanic persons reported as other or multiple races.

$\S \S$ Among sampled patients, COVID-NET collects data on the primary reason for admission to differentiate hospitalizations of patients with laboratory-confirmed SARS-CoV-2 infection who are likely admitted primarily for COVID-19 illness rather than for other reasons. During chart review, if the surveillance officer finds that the chief complaint or history of present illness mentions fever or respiratory illness, COVID-19-like illness, or suspected COVID-19, then the case is categorized as COVID-19-related illness as the primary reason for admission. Reasons for admission that are likely primarily not related to COVID-19 include the following categories: obstetrics/labor and delivery, inpatient surgery or procedures, psychiatric admission requiring acute medical care, trauma, other, or unknown. Reasons categorized as "other" are reviewed by two physicians to determine whether the admission is likely COVID-19 related.

१ी COVID-19-related symptoms included respiratory symptoms (congestion or runny nose, cough, hemoptysis or bloody sputum, shortness of breath or respiratory distress, sore throat, upper respiratory infection, influenza-like illness, and wheezing) and nonrespiratory symptoms (abdominal pain, altered mental status or confusion, anosmia or decreased smell, chest pain, conjunctivitis, diarrhea, dysgeusia or decreased taste, fatigue, fever or chills, headache, muscle aches or myalgias, nausea or vomiting, rash, and seizures), and among those aged $<2$ years, included apnea, cyanosis, decreased vocalization or stridor, dehydration, hypothermia, inability to eat or poor feeding, and lethargy. Symptoms are abstracted from the medical chart and might not be complete.

*** ICU admission and invasive mechanical ventilation are not mutually exclusive categories, and patients could have received both.

${ }^{+\dagger \dagger}$ Fully vaccinated adolescents with COVID-19-associated hospitalizations were defined as those who had received the final dose in their primary series $\geq 14$ days before receiving a positive SARS-CoV-2 test result associated with their hospitalization. Adolescents who received only 1 vaccine dose $\geq 14$ days before the SARS-CoV- 2 test date or had received a single dose of vaccine $<14$ days before the positive SARS-CoV- 2 test results were considered partially vaccinated; they were not included in rates and were grouped with unvaccinated adolescents in other analyses. COVID-NET sites, through agreements with state health departments and other partners, collect COVID-19 vaccination information on COVID-19-associated hospitalizations through state-based vaccine registries. When possible, sites collect COVID-19 vaccination status on all persons with COVID-19 cases who are hospitalized, including the number of vaccine doses received, the vaccine product, and dates of vaccination administration.
\end{abstract}

definition for being fully vaccinated until December 7, 2021, vaccination among this age group was not considered in this study. However, vaccinations could have affected hospitalization rates during the Omicron period. Further, boosters among adolescents aged 12-17 years could not be examined because the recommendation was so recent. Finally, the COVID-NET catchment areas include approximately $10 \%$ of the U.S. population; thus, these findings might not be generalizable to the entire United States.

Coinciding with emerging predominance of the Omicron variant, rates of COVID-19-associated hospitalization among children and adolescents increased rapidly during the last 2 weeks of December 2021, especially among those aged 0-4 years. Moreover, among adolescents, hospitalization rates were higher among those who were unvaccinated. Vaccination of eligible persons, in addition to other prevention strategies such as masking, are critical to reducing the incidence of severe COVID-19 among children and adolescents. 99999 All persons who are eligible for vaccination should receive and stay up to

\footnotetext{
9999 https:/www.cdc.gov/coronavirus/2019-ncov/variants/omicron-variant.html
}

date with COVID-19 vaccines to reduce the risk for severe disease for themselves and others with whom they come into contact, including children who are currently too young to be vaccinated.******

$\overline{* * * * * * h t t p s: / / w w w . c d c . g o v / c o r o n a v i r u s / 2019-n c o v / v a c c i n e s / s t a y-u p-t o-d a t e . h t m l ~}$

\section{Acknowledgments}

Joelle Nadle, Sherry Quach, Jeremy Roland, Gretchen Rothrock, California Emerging Infections Program, Oakland, California; Rachel Herlihy, Madelyn Lensing, Jordan Surgnier, Millen Tsegaye, Colorado Department of Public Health and Environment; Ann Basting, Tessa Carter, Daewi Kim, Julie Plano, Connecticut Emerging Infections Program, Yale School of Public Health; Marina Bruck, Rayna Ceaser, Gracie Chambers, Taylor Eisenstein, Sabrina Hendrick, Johanna Hernandez, Asmith Joseph, Grayson Kallas, Stephanie Lehman, Jana Manning, Annabel Patterson, Allison Roebling, Suzanne Segler, Chandler Surell, Hope Wilson, School of Medicine, Emory University, Georgia Emerging Infections Program, Georgia Department of Public Health, Veterans Affairs Medical Center, Foundation for Atlanta Veterans Education and Research, Atlanta, Georgia; Chloe Brown, Jim Collins, Shannon Johnson, Sue Kim, Alexander Kohrman, Lauren Leegwater, Sierra Peguies-Khan, 
TABLE 2. Demographic and clinical characteristics and outcomes among fully vaccinated* and unvaccinated adolescents aged $12-17$ years with laboratory-confirmed COVID-19-associated hospitalizations, ${ }^{\dagger}$ by date of admission — COVID-NET, 14 states, ${ }^{\S}$ July 1 -December 31 , 2021

\begin{tabular}{|c|c|c|c|c|c|c|c|}
\hline \multirow[b]{4}{*}{ Characteristic } & \multicolumn{7}{|c|}{ No. of hospitalized adolescents (\%) } \\
\hline & Unvaccinated & Vaccinated & \multirow[b]{3}{*}{ p-value ${ }^{q}$} & \multicolumn{2}{|c|}{ Unvaccinated } & \multicolumn{2}{|c|}{ Vaccinated } \\
\hline & \multicolumn{2}{|c|}{ Total } & & & & & \\
\hline & \multicolumn{2}{|c|}{ Jul 1-Dec 31} & & Jul 1-Dec 18 & Dec 19-31 & Jul 1-Dec 18 & Dec 19-31 \\
\hline Total & $647(100.0)^{* *}$ & $71(100.0)^{* *}$ & - & $584(90.2)^{* *}$ & $63(9.8)^{* *}$ & $53(74.6)^{* *}$ & $18(25.4)^{* *}$ \\
\hline Age, yrs, median (IQR) & $15(14-16)$ & $15(14-16)$ & 0.75 & $15(14-17)$ & $14(13-16)$ & $15(14-16)$ & $15(14-16)$ \\
\hline $\begin{array}{l}\text { Sex } \\
\text { Male } \\
\text { Female }\end{array}$ & $\begin{array}{l}298(46.0) \\
349(54.0)\end{array}$ & $\begin{array}{l}25(35.2) \\
46(64.8)\end{array}$ & 0.08 & $\begin{array}{l}266(45.5) \\
318(54.5)\end{array}$ & $\begin{array}{l}32(50.8) \\
31(49.2)\end{array}$ & $\begin{array}{l}20(37.7) \\
33(62.3)\end{array}$ & $\begin{array}{r}5(27.7) \\
13(72.3)\end{array}$ \\
\hline $\begin{array}{l}\text { Race and ethnicity }{ }^{\dagger \dagger} \\
\text { Hispanic } \\
\text { Black, non-Hispanic } \\
\text { White, non-Hispanic } \\
\text { Asian or Pacific Islander, non-Hispanic } \\
\text { All other races§§ } \\
\text { Unknown race and ethnicity }\end{array}$ & $\begin{array}{r}148(22.9) \\
274(42.4) \\
162(25.0) \\
14(2.2) \\
20(3.1) \\
29(4.5)\end{array}$ & $\begin{array}{r}14(19.7) \\
17(24.0) \\
32(45.0) \\
1(1.4) \\
2(2.8) \\
5(7.1)\end{array}$ & 0.006 & $\begin{array}{r}136(23.3) \\
240(41.1) \\
154(26.4) \\
10(1.7) \\
19(3.3) \\
25(4.3)\end{array}$ & $\begin{array}{r}12(19.0) \\
34(53.9) \\
8(12.7) \\
4(6.4) \\
1(1.6) \\
4(6.4)\end{array}$ & $\begin{array}{r}12(22.6) \\
11(20.8) \\
25(47.1) \\
1(1.9) \\
1(1.9) \\
3(5.7)\end{array}$ & $\begin{array}{r}2(11.1) \\
6(33.4) \\
7(38.8) \\
0(-) \\
1(5.5) \\
2(11.2)\end{array}$ \\
\hline $\begin{array}{l}\text { Primary reason for admission }{ }^{\text {१ी }} \\
\text { Likely related to COVID-19 } \\
\text { Obstetrics } \\
\text { Inpatient surgery } \\
\text { Psychiatric admission requiring medical care } \\
\text { Trauma } \\
\text { Other reason } \\
\text { Unknown reason }\end{array}$ & $\begin{array}{r}454(70.3) \\
40(6.2) \\
15(2.3) \\
79(12.2) \\
40(6.2) \\
16(2.5) \\
2(0.3)\end{array}$ & $\begin{array}{r}29(40.8) \\
0(-) \\
7(9.8) \\
27(38.1) \\
4(5.6) \\
4(5.6) \\
0(-)\end{array}$ & $<0.001$ & $\begin{array}{r}413(70.8) \\
36(6.2) \\
13(2.2) \\
72(12.4) \\
35(6.0) \\
12(2.1) \\
2(0.3)\end{array}$ & $\begin{array}{r}41(65.0) \\
4(6.4) \\
2(3.2) \\
7(11.1) \\
5(8.0) \\
4(6.3) \\
0(-)\end{array}$ & $\begin{array}{r}19(35.8) \\
0(-) \\
5(9.4) \\
24(45.3) \\
3(5.7) \\
2(3.8) \\
0(-)\end{array}$ & $\begin{array}{r}10(55.5) \\
0(-) \\
2(11.1) \\
3(16.8) \\
1(5.5) \\
2(11.1) \\
0(-)\end{array}$ \\
\hline $\begin{array}{l}\text { COVID-19-related symptoms at admission* } \\
\text { Yes } \\
\text { No }\end{array}$ & $\begin{array}{l}\text { ** } \\
536(83.0) \\
110(17.0)\end{array}$ & $\begin{array}{l}53(74.6) \\
18(25.4)\end{array}$ & 0.08 & $\begin{array}{r}487(83.4) \\
97(16.6)\end{array}$ & $\begin{array}{l}49(79.0) \\
13(21.0)\end{array}$ & $\begin{array}{l}37(69.8) \\
16(30.2)\end{array}$ & $\begin{array}{r}16(88.9) \\
2(11.1)\end{array}$ \\
\hline $\begin{array}{l}\text { Hospitalization outcomes } \\
\text { Length of hospital stay, days, median (IQR) } \\
\text { ICU admission+†† } \\
\text { Invasive mechanical ventilationt†† } \\
\text { In-hospital death }\end{array}$ & $\begin{array}{r}4(2-7) \\
196(30.3) \\
42(6.5) \\
5(0.8) \\
\end{array}$ & $\begin{array}{r}3(1-8) \\
11(15.5) \\
6(8.4) \\
2(2.8) \\
\end{array}$ & $\begin{array}{l}0.55 \\
0.009 \\
0.54 \\
0.10 \\
\end{array}$ & $\begin{array}{r}4(2-6.5) \\
184(31.6) \\
41(7.1) \\
5(0.9) \\
\end{array}$ & $\begin{array}{r}4(2-8) \\
12(19.1) \\
1(1.6) \\
0(-) \\
\end{array}$ & $\begin{array}{r}3(2-9) \\
8(15.1) \\
5(9.4) \\
2(3.8) \\
\end{array}$ & $\begin{array}{r}3(1-5) \\
3(16.6) \\
1(5.5) \\
0(-) \\
\end{array}$ \\
\hline
\end{tabular}

See table footnotes on the next page.

Libby Reeg, Michigan Department of Health and Human Services; Alison Babb, Richard Danila, Kristen Ehresmann, Jake Garfin, Jennifer Gilbertson, Grace Hernandez, Melissa McMahon, Kieu My Phi, Jill Reaney, Sara Vetter, Xiong Wang, Minnesota Department of Health; Cory Cline, Melissa Judson, Sunshine Martinez, Florent Nkouaga, Jasmyn Sanchez, Daniel Sosin, New Mexico Department of Health; Kathy M. Angeles, Molly Bleecker, Sarah Shrum Davis, Nancy Eisenberg, Sarah A. Khanlian, Sarah Lathrop, Wickliffe Omondi, Mayvilynne Poblete, Dominic Rudin, Yadira SalazarSanchez, New Mexico Emerging Infections Program; Jennifer Akpo, Celina Chavez, Yassir Talha, Alesia Reed, CDC Foundation, New Mexico Department of Health; Kerianne Engesser, Suzanne McGuire, New York State Department of Health; Christina Felsen, Maria Gaitan, Christine Long, Thomas Peer, University of Rochester School of Medicine and Dentistry; Julie Freshwater, Denise IngabireSmith, Ann Salvator, Rebekah Sutter, Ohio Department of Health; Kathy Billings, Katie Dyer, Anise Elie, Gail Hughett, Karen Leib, Terri McMinn, Danielle Ndi, Manideepthi Pemmaraju, Emmanuel Sackey, Vanderbilt University Medical Center; Ian Buchta, Amanda Carter, Melanie Crossland, Andrew Haraghey, Mary Hill, Laine McCullough, Jake Ortega, Tyler Riedesel, Caitlin Shaw, Ashley

\section{Summary}

What is already known about this topic?

COVID-19 can cause severe illness in children and adolescents.

What is added by this report?

Coinciding with increased circulation of the Omicron variant, COVID-19-associated hospitalization rates among children and adolescents aged 0-17 years increased rapidly in late December 2021, especially among children aged 0-4 years who are not yet eligible for vaccination. Throughout the periods of Delta and Omicron predominance, hospitalization rates remained lower among fully vaccinated adolescents aged 12-17 years than among unvaccinated adolescents.

What are the implications for public health practice? Strategies to prevent COVID-19 among children and adolescents, including vaccination of eligible persons, are critical.

Swain, Salt Lake County Health Department, Salt Lake City, Utah; Rainy Henry, Sonja Mali Nti-Berko, Robert W. Pinner, Alvin Shultz, CDC; Mimi Huynh, Council of State and Territorial Epidemiologists. 
TABLE 2. (Continued) Demographic and clinical characteristics and outcomes among fully vaccinated* and unvaccinated adolescents aged $12-17$ years with laboratory-confirmed COVID-19-associated hospitalizations, ${ }^{\dagger}$ by date of admission - COVID-NET, 14 states, ${ }^{\S}$ July 1-December 31,2021

\begin{abstract}
Abbreviations: COVID-NET = Coronavirus Disease 2019-Associated Hospitalization Surveillance Network; ICU = intensive care unit.
* Fully vaccinated adolescents with COVID-19-associated hospitalizations were defined as those who had received the final dose in their primary series $\geq 14$ days before receiving a positive SARS-CoV-2 test result associated with their hospitalization. Adolescents who received only 1 vaccine dose $\geq 14$ days before the SARS-CoV-2 test date or had received a single dose of vaccine $<14$ days before the positive SARS-CoV- 2 test results were considered partially vaccinated; they were not included in rates and were grouped with unvaccinated adolescents in other analyses. COVID-NET sites, through agreements with state health departments and other partners, collect COVID-19 vaccination information on COVID-19-associated hospitalizations through state-based vaccine registries. When possible, sites collect COVID-19 vaccination status on all persons with COVID-19 cases who are hospitalized, including the number of vaccine doses received, the vaccine product, and dates of vaccination administration.

† Data are from a weighted sample of hospitalized children and adolescents with completed medical record abstractions. Sample sizes presented are unweighted with weighted percentages.

\$Includes persons admitted to a hospital with an admission date during July 1-December 31, 2021. Maryland contributed data through November 26, 2021. Counties included in COVID-NET surveillance: California (Alameda, Contra Costa, and San Francisco counties); Colorado (Adams, Arapahoe, Denver, Douglas, and Jefferson counties); Connecticut (Middlesex and New Haven counties); Georgia (Clayton, Cobb, DeKalb, Douglas, Fulton, Gwinnett, Newton, and Rockdale counties); lowa (one county represented); Maryland (Allegany, Anne Arundel, Baltimore, Baltimore City, Calvert, Caroline, Carroll, Cecil, Charles, Dorchester, Frederick, Garrett, Harford, Howard, Kent, Montgomery, Prince George's, Queen Anne's, St. Mary's, Somerset, Talbot, Washington, Wicomico, and Worcester counties); Michigan (Clinton, Eaton, Genesee, Ingham, and Washtenaw counties); Minnesota (Anoka, Carver, Dakota, Hennepin, Ramsey, Scott, and Washington counties); New Mexico (Bernalillo, Chaves, Doña Ana, Grant, Luna, San Juan, and Santa Fe counties); New York (Albany, Columbia, Genesee, Greene, Livingston, Monroe, Montgomery, Ontario, Orleans, Rensselaer, Saratoga, Schenectady, Schoharie, Wayne, and Yates counties); Ohio (Delaware, Fairfield, Franklin, Hocking, Licking, Madison, Morrow, Perry, Pickaway, and Union counties); Oregon (Clackamas, Multnomah, and Washington counties); Tennessee (Cheatham, Davidson, Dickson, Robertson, Rutherford, Sumner, Williamson, and Wilson counties); and Utah (Salt Lake County).

I Proportions between vaccinated and unvaccinated adolescents were compared with chi-square tests or Fisher's exact tests (as appropriate), and medians were compared with the Wilcoxon rank sum test; $p$-values $<0.05$ were considered statistically significant.

** Data are missing for $<5 \%$ of observations for all variables.

t+ If ethnicity was unknown, non-Hispanic ethnicity was assumed.

$\S \S$ Includes non-Hispanic persons reported as other or multiple races.

Iी Among sampled patients, COVID-NET collects data on the primary reason for admission to differentiate hospitalizations of patients with laboratory-confirmed SARS-CoV-2 infection who are likely admitted primarily for COVID-19 illness rather than for other reasons. During chart review, if the surveillance officer finds that the chief complaint or history of present illness mentions fever or respiratory illness, COVID-19-like illness, or suspected COVID-19, then the case is categorized as COVID-19-related illness as the primary reason for admission. Reasons for admission that are likely primarily not related to COVID-19 include the following categories: obstetrics/labor and delivery, inpatient surgery or procedures, psychiatric admission requiring acute medical care, trauma, other, or unknown. Reasons categorized as "other" are reviewed by two physicians to determine whether the admission is likely COVID-19 related.

*** COVID-19-related symptoms included respiratory symptoms (congestion or runny nose, cough, hemoptysis or bloody sputum, shortness of breath or respiratory distress, sore throat, upper respiratory infection, influenza-like illness, and wheezing) and nonrespiratory symptoms (abdominal pain, altered mental status or confusion, anosmia or decreased smell, chest pain, conjunctivitis, diarrhea, dysgeusia or decreased taste, fatigue, fever or chills, headache, muscle aches or myalgias, nausea or vomiting, rash, and seizures), and among those aged $<2$ years, included apnea, cyanosis, decreased vocalization or stridor, dehydration, hypothermia, inability to eat or poor feeding, and lethargy. Symptoms are abstracted from the medical chart and might not be complete.

${ }^{t+\dagger}$ ICU admission and invasive mechanical ventilation are not mutually exclusive categories, and patients could have received both.
\end{abstract}

Corresponding author: Kristin J. Marks; KJMarks@cdc.gov.

${ }^{1}$ CDC COVID-19 Emergency Response Team; ${ }^{2}$ Epidemic Intelligence Service, CDC; ${ }^{3}$ General Dynamics Information Technology, Atlanta, Georgia; ${ }^{4}$ California Emerging Infections Program, Oakland, California; ${ }^{5}$ Career Epidemiology Field Officer Program, CDC; ${ }^{6}$ Colorado Department of Public Health and Environment; ${ }^{7}$ Connecticut Emerging Infections Program, Yale School of Public Health, New Haven, Connecticut; ${ }^{8}$ Emory University School of Medicine, Atlanta, Georgia; ${ }^{9}$ Georgia Emerging Infections Program, Georgia Department of Public Health; ${ }^{10}$ Atlanta Veterans Affairs Medical Center, Atlanta, Georgia; ${ }^{11}$ Iowa Department of Public Health; ${ }^{12}$ Michigan Department of Health and Human Services; ${ }^{13}$ Minnesota Department of Health; ${ }^{14} \mathrm{New}$ Mexico Department of Health; ${ }^{15} \mathrm{New}$ York State Department of Health; ${ }^{16}$ University of Rochester School of Medicine and Dentistry, Rochester, New York; ${ }^{17}$ Ohio Department of Health; ${ }^{18}$ Public Health Division, Oregon Health Authority; ${ }^{19}$ Vanderbilt University Medical Center, Nashville, Tennessee; ${ }^{20}$ Salt Lake County Health Department, Salt Lake City, Utah.

\section{COVID-NET Surveillance Team}

Arthur Reingold, University of California, Berkeley, Berkeley, California; Nisha Alden, Colorado Department of Public Health and Environment; Breanna Kawasaki, Colorado Department of Public Health and Environment; Maria Correa, Connecticut Emerging Infections Program, Yale School of Public Health, New Haven, Connecticut; Carol Lyons, Connecticut Emerging Infections
Program, Yale School of Public Health, New Haven, Connecticut; Emily Fawcett, Georgia Emerging Infections Program, Georgia Department of Public Health, Atlanta, Georgia, Veterans Affairs Medical Center, Atlanta, Georgia, Foundation for Atlanta Veterans Education and Research, Decatur, Georgia; Katelyn Ward, Georgia Emerging Infections Program, Georgia Department of Public Health, Atlanta, Georgia, Division of Infectious Diseases, Emory University School of Medicine, Atlanta, Georgia; Kayla Bilski, Minnesota Department of Health; Erica Bye, Minnesota Department of Health; Emily B. Hancock, University of New Mexico Health Sciences Center, New Mexico Emerging Infections Program, Albuquerque, New Mexico; Murtada Khalifa, CDC Foundation, Atlanta, Georgia, New Mexico Department of Health; Adam Rowe, New York State Department of Health; Nancy Spina, New York State Department of Health; Virginia Cafferky, University of Rochester School of Medicine and Dentistry, Rochester, New York; Kevin Popham, University of Rochester School of Medicine and Dentistry, Rochester, New York; Sam Hawkins, Public Health Division; Oregon Health Authority; Tiffanie Markus, Vanderbilt University Medical Center, Nashville, Tennessee; Keegan McCaffrey, Utah Department of Health; Andrea Price, Salt Lake County Health Department, Salt Lake City, Utah. 
All authors have completed and submitted the International Committee of Medical Journal Editors form for disclosure of potential conflicts of interest. Evan J. Anderson reports grants from Pfizer, Merck, PaxVax, Micron, Sanofi-Pasteur, Janssen, MedImmune, and GlaxoSmithKline; personal fees from Pfizer, Medscape, Kentucky Bioprocessing, Inc., Sanofi-Pasteur, and Janssen, outside the submitted work; and institutional funding from the National Institutes of Health to conduct clinical trials of Moderna and Janssen COVID-19 vaccines. Laurie M. Billing, Eli Shiltz, Andy Weigel, Justin Henderson, Val Tellez Nunez, and Andrea George report grants from the Council of State and Territorial Epidemiologists during the conduct of the study. Ruth Lynfield reports editorial payments from the American Academy of Pediatrics Red Book (Committee on Infectious Diseases), which were donated to the Minnesota Department of Health. No other potential conflicts of interest were disclosed.

\section{References}

1. CDC COVID-19 Response Team. SARS-CoV-2 B.1.1.529 (Omicron) variant-United States, December 1-8, 2021. MMWR Morb Mortal Wkly Rep 2021;70:1731-4. PMID:34914670 https://doi.org/10.15585/ mmwr.mm7050e1

2. Delahoy MJ, Ujamaa D, Whitaker M, et al.; COVID-NET Surveillance Team. Hospitalizations associated with COVID-19 among children and adolescents-COVID-NET, 14 states, March 1, 2020-August 14, 2021. MMWR Morb Mortal Wkly Rep 2021;70:1255-60. PMID:34499627 https://doi.org/10.15585/mmwr.mm7036e2

3. Woodruff RC, Campbell AP, Taylor CA, et al. Risk factors for severe COVID-19 in children. Pediatrics 2021;e2021053418. PMID:34935038 https://doi.org/10.1542/peds.2021-053418
4. Feldstein LR, Rose EB, Horwitz SM, et al.; Overcoming COVID-19 Investigators; CDC COVID-19 Response Team. Multisystem inflammatory syndrome in U.S. children and adolescents. N Engl J Med 2020;383:334-46. PMID:32598831 https://doi.org/10.1056/ NEJMoa2021680

5. Barrett CE, Koyama AK, Alvarez P, et al. Risk for newly diagnosed diabetes $>30$ days after SARS-CoV-2 infection among persons aged $<18$ yearsUnited States, March 1, 2020-June 28, 2021. MMWR Morb Mortal Wkly Rep 2022;71:59-65. PMID:35025851 https://doi.org/10.15585/ mmwr.mm7102e 2

6. Olson SM, Newhams MM, Halasa NB, et al.; Overcoming Covid-19 Investigators. Effectiveness of BNT162b2 vaccine against critical Covid-19 in adolescents. N Engl J Med 2022;NEJMoa2117995. PMID:35021004 https://doi.org/10.1056/NEJMoa2117995

7. Iuliano AD, Brunkard JM, Boehmer TK, et al. Trends in disease severity and health care utilization during the early Omicron variant period compared with previous SARS-CoV-2 high transmission periods-United States, December 2020-January 2022. MMWR Morb Mortal Wkly Rep 2022;71:146-52. PMID:35085225 https://doi.org/10.15585/mmwr. $\mathrm{mm} 7104 \mathrm{e} 4$

8. Havers FP, Whitaker M, Self JL, et al.; COVID-NET Surveillance Team. Hospitalization of adolescents aged 12-17 years with laboratoryconfirmed COVID-19-COVID-NET, 14 states, March 1, 2020April 24, 2021. MMWR Morb Mortal Wkly Rep 2021;70:851-7. PMID:34111061 https://doi.org/10.15585/mmwr.mm7023e1

9. Wanga V, Gerdes ME, Shi DS, et al. Characteristics and clinical outcomes of children and adolescents aged $<18$ years hospitalized with COVID-19six hospitals, United States, July-August 2021. MMWR Morb Mortal Wkly Rep 2021;70:1766-72. PMID:34968374 https://doi.org/10.15585/ mmwr.mm705152a3 